\title{
Coordinated Regulation of Ammonium Assimilation and Carbon Catabolism by Glyoxylate in Saccharomyces cerevisiae
}

\author{
By ALICIA GONZÁlEZ,* LAURA RODRÍGUEZ, \\ JORGE FOLCH, MARIO SOBERÓN AND HIRAM OLIVERA \\ Centro de Investigación sobre Fijación de Nitrógeno, UNAM, Departamento de Biologia \\ Molecular de Plantas, Apartado Postal 565-A, Cuernavaca, Morelos, México
}

(Received 29 December 1986; revised 24 March 1987)

\begin{abstract}
The activities of citrate synthase (EC 4.1.3.7) and NADP+-dependent glutamate dehydrogenase (GDH) (EC 1 .4 .1 .4) of Saccharomyces cerevisiae were inhibited in vitro by glyoxylate. In the presence of glyoxylate, pyruvate and glyoxylate pools increased, suggesting that glyoxylate was efficiently transported and catabolized. Pyruvate accumulation also indicates that citrate synthase was inhibited. A decrease in the glutamate pool was also observed under these conditions. This can be attributed to an increased transamination rate and to the inhibitory effect of glyoxylate on NADP ${ }^{+}$-dependent GDH. Furthermore, the increase in the ammonium pool in the presence of glyoxylate suggests that $\mathrm{NADP}^{+}$-dependent GDH was being inhibited in vivo, since the activity of glutamine synthetase did not decrease under these conditions. We propose that the inhibition of both citrate synthase and NADP ${ }^{+}$-dependent GDH could form part of a mechanism that regulates the internal 2-oxoglutarate concentration.
\end{abstract}

\section{INTRODUCTION}

$\mathrm{NADP}^{+}$-dependent glutamate dehydrogenase $(\mathrm{GDH})$ catalyses the synthesis of glutamate from ammonium and 2-oxoglutarate (Holzer \& Schneider, 1957). Ammonium is derived from the metabolism of nitrogenous compounds, or from the medium, and 2-oxoglutarate from carbon metabolism. Thus, ammonium assimilation constitutes the metabolic process in which carbon and nitrogen metabolism meet, and NADP ${ }^{+}$-dependent GDH is the enzyme that links these two metabolic pathways. It has been proposed that in Saccharomyces cerevisiae, 2oxoglutarate, or some other intermediate of the tricarboxylic acid cycle, modulates NADP+ dependent GDH activity (González et al., 1985). Furthermore, it could be expected that fluctuations in the intracellular levels of intermediates of the cycle could in turn regulate the activity of the NADP ${ }^{+}$-dependent GDH. Thus, regulation of the tricarboxylic acid cycle could have a direct effect on $\mathrm{NADP}^{+}$-dependent $\mathrm{GDH}$ activity and therefore on ammonium assimilation. This mechanism may constitute a regulatory link between these two metabolic pathways, which could determine the amount of 2-oxoglutarate and ammonium that can be assimilated in a given physiological condition.

It has been shown that cells of Escherichia coli growing on glyoxylate contain much lower levels of citrate synthase than similar cells growing on acetate (Kornberg, 1966), suggesting that this compound could modulate the operation of the tricarboxylic acid cycle by regulating the levels of citrate synthase. Furthermore, since the metabolism of glyoxylate through the dicarboxylic acid cycle does not produce 2-oxoglutarate, it would be expected that in the presence of glyoxylate, intracellular 2-oxoglutarate levels would be decreased, and that this would affect the activity of the $\mathrm{NADP}^{+}$-dependent GDH and thus ammonium assimilation. We

Abbreviations: GDH, glutamate dehydrogenase; MM, minimal medium. 
studied the effect of glyoxylate on citrate synthase and NADP+-dependent GDH in S. cerevisiae in order to determine whether this 2-oxoacid could play a role in the regulation of carbon and nitrogen metabolism.

\section{METHODS}

Strain and growth conditions. The wild-type strain S288C (MAT $\alpha$ mal gal2) was obtained from the Cold Spring Harbor Laboratory, NY, USA. Cells were routinely grown on minimal medium (MM) containing salts, trace elements and vitamins following the formula of Difco Yeast Nitrogen Base. Glucose $(2 \%, w / v)$ was used as carbon source and $40 \mathrm{mM}-\left(\mathrm{NH}_{4}\right)_{2} \mathrm{SO}_{4}$ as nitrogen source. Glyoxylate or pyruvate were added to the cultures, as appropriate, as aqueous autoclaved solutions. The cultures were incubated at $30^{\circ} \mathrm{C}$ with agitation. Cells were grown on YPD medium comprising $1 \%(w / v)$ yeast extract (Difco), $2 \%(w / v)$ peptone (Difco) and $2 \%(w / v)$ glucose, until they reached stationary phase; they were then washed and resuspended in MM. These suspensions were used to inoculate cultures to an $\mathrm{OD}_{650}$ of 0.05 .

Extraction and determination of intracellular metabolites. For the estimation of 2-oxoglutarate, pyruvate and glyoxylate, extracts were prepared as described by Kang et al. (1982). Ammonium was extracted by the method of Tempest et al. (1970) and Tachiki et al. (1981). The concentrations of ammonium and 2-oxoglutarate were determined with beef GDH by following NADH oxidation at $340 \mathrm{~nm}$ (Dubois et al., 1974). Pyruvate concentration was determined with beef lactate dehydrogenase by following NADH oxidation at $340 \mathrm{~nm}$ (Bergmeyer, 1963). Glyoxylate was determined by the method of Tribels \& Vogel (1966).

Amino acids were extracted and determined as described by González et al. (1983). For enzyme determinations, soluble extracts were prepared by grinding whole cells, suspended in their corresponding extraction buffer, with glass beads in a Braun cell disruptor. Citrate synthase (EC 4.1.3.7), NADP+-dependent GDH (EC 1.4.1.4) and glutamine synthetase (EC 6.3.1 .2) were assayed by the methods of Parvin (1969), Doherty (1970) and Ferguson \& Sims (1974) respectively. Protein was determined by the Lowry method, using bovine serum albumin as standard.

Chemicals. All 2-oxoacids, beef GHD, beef lactate dehydrogenase and bovine serum albumin were obtained from Sigma.

\section{RESULTS AND DISCUSSION}

\section{Effect of glyoxylate on citrate synthase activity}

Citrate synthase from cells grown on $\mathrm{MM}$ for $6 \mathrm{~h}$ was inhibited by glyoxylate but not pyruvate (Fig. 1). In order to study whether the inhibition of citrate synthase could alter the carbon flow through the tricarboxylic acid cycle we determined the intracellular content of 2-oxoglutarate. In glyoxylate-treated cells 2-oxoglutarate levels were 4-fold higher than in cells incubated in MM without glyoxylate; glyoxylate-treated cells had almost 30 -fold higher glyoxylate levels than those from the untreated culture (Table 1). This could be explained by assuming that the utilization of 2-oxoglutarate by $\mathrm{NADP}^{+}$-dependent $\mathrm{GDH}$ had diminished. It has been proposed that the catabolism of glyoxylate through the dicarboxylic acid cycle can result in the production of pyruvate (Kornberg, 1966). If this were the case, the increase in the pyruvate pools could shift the endogenous glutamate pools towards transamination, which would result in increased levels of 2-oxoglutarate. The pyruvate level in glyoxylate-treated cells was 14-fold higher than cells in MM (Table 1).

\section{Effect of glyoxylate on nitrogen assimilation}

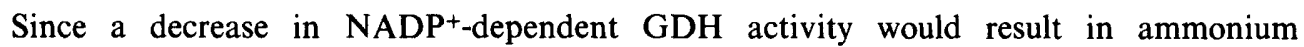
accumulation, we measured the amount of ammonium present in glyoxylate-treated and untreated cells. Ammonium levels were $2 \cdot 8$-fold higher in glyoxylate-treated cells (Fig. $2 a$ ). This accumulation could be attributed to a decrease in ammonium assimilation through NADP+dependent GDH or through glutamine synthetase. Since the activity of the latter enzyme reached values 4-fold higher in glyoxylate-treated cells than in control cells (Fig. 3), ammonium accumulation can only be the result of decreased $\mathrm{NADP}^{+}$-dependent GDH activity. Furthermore, the rise in glutamine synthetase activity (Fig. 3) was accompanied by a decrease in the ammonium pool that had accumulated, suggesting that under these conditions ammonium assimilation proceeded through glutamine synthetase. As a control, we determined the effect of the addition of pyruvate instead of glyoxylate. The ammonium pool in the pyruvate-treated cells was very similar to that in the control culture (Fig. 2b). 2-Oxoglutarate levels were similar in cells 


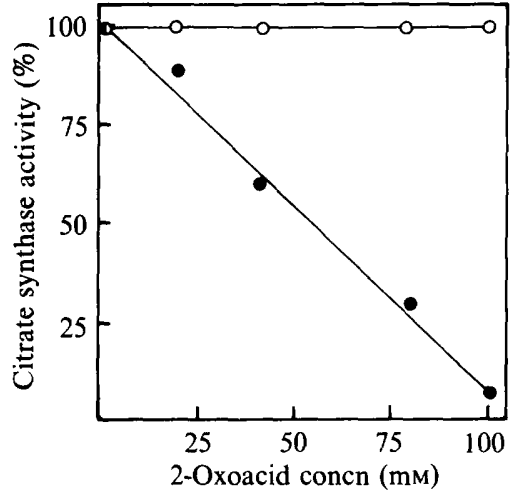

Fig. 1

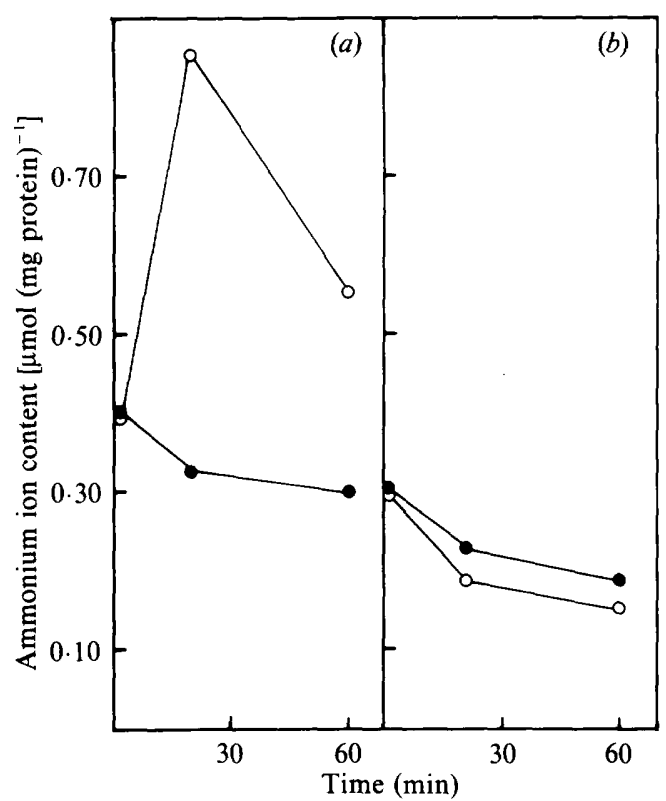

Fig. 2

Fig. 1. Effect of glyoxylate $(\bullet)$ and pyruvate $(O)$ on citrate synthase. Activity was assayed in vitro. The activity corresponding to $100 \%$ was $4.7 \mathrm{U}$ (mg protein $)^{-1}$. Results shown are representative of at least three determinations; variation was 5 to $10 \%$.

Fig. 2. Effect of glyoxylate and pyruvate on ammonium ion pools. (a) Cells were grown in $\mathrm{MM}$ for $2 \mathrm{~h}$; the culture was then divided and $20 \mathrm{mM}$-glyoxylate $(O)$ was added to one half; the rest of the culture was left untreated (৩). Ammonium pools were then determined. (b) Cells were treated as in $(a)$, but half the culture was treated with $20 \mathrm{~mm}$-pyruvate $(O)$ instead of glyoxylate; the other half was again untreated (๑). Results shown are representative of at least three determinations; variation was 5 to $10 \%$.

\section{Table 1. Intracellular pools of 2-oxoacids in S. cerevisiae treated with glyoxylate or pyruvate}

Cells were grown in $\mathrm{MM}$ for $2 \mathrm{~h}$; the culture was then divided, and half was treated with $20 \mathrm{mM}$ glyoxylate; the other half was left untreated. In a separate experiment cells were treated with $20 \mathrm{~mm}$ pyruvate instead of glyoxylate. After $20 \mathrm{~min}$, samples were removed from both cultures, the cells were washed with MM and extracts were prepared and assayed as described in Methods. Results shown are means of three determinations; values in parentheses show variance of the mean.

\begin{tabular}{|c|c|c|c|}
\hline \multirow[b]{2}{*}{ 2-Oxoacid } & \multicolumn{3}{|c|}{ 2-Oxoacid content $\left[\mathrm{nmol}(\mathrm{mg} \text { protein })^{-1}\right]$} \\
\hline & MM & MM + glyoxylate & $\mathbf{M M}+$ pyruvate \\
\hline Glyoxylate & $11.61 \quad(1.43)$ & $326 \cdot 60(288 \cdot 80)$ & $(0.66)$ \\
\hline Pyruvate & $51.44(10 \cdot 52)$ & $758 \cdot 30(972 \cdot 20)$ & $325.00(416.67)$ \\
\hline 2-Oxoglutarate & $9.20 \quad(0.24)$ & $39.43 \quad(11.61)$ & $(0 \cdot 33)$ \\
\hline
\end{tabular}

in $\mathrm{MM}$ with and without pyruvate (Table 1). These data indicate that glyoxylate negatively modulates $\mathrm{NADP}^{+}$-dependent $\mathrm{GDH}$, whereas pyruvate does not. Glyoxylate inhibited $\mathrm{NADP}^{+}$-dependent GDH activity in vitro while pyruvate did not (Fig. 4). This suggests that inhibition of $\mathrm{NADP}^{+}$-dependent GDH by glyoxylate provides a mechanism which compensates for the decrease in carbon flow due to the inhibition of citrate synthase by glyoxylate. This mechanism could play a role when poor carbon sources such as acetate are provided, since in these conditions, the activity of 2-oxoglutarate should be strictly regulated. In this regard, it has been reported that the enzymes which participate in glyoxylate synthesis are induced in acetate- 


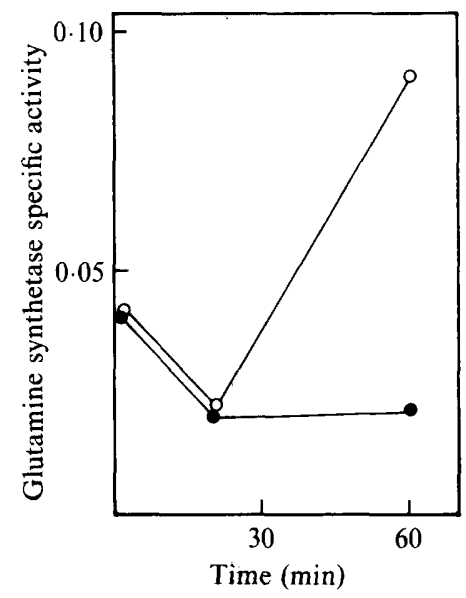

Fig. 3

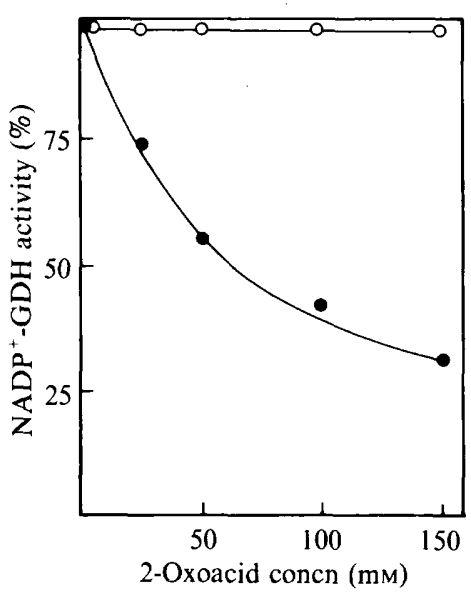

Fig. 4

Fig. 3. Effect of glyoxylate on glutamine synthetase specific activity. Cells were grown in MM for $2 \mathrm{~h}$; the culture was then divided and $20 \mathrm{~mm}$-glyoxylate $(O)$ was added to one half; the rest of the culture was left untreated (-). Glutamine synthetase activity was then determined. Specific activity is expressed as $\mu$ mol $\gamma$-glutamyl hydroxamate produced $\min ^{-1}(\mathrm{mg} \text { protein })^{-1}$ at $30^{\circ} \mathrm{C}$. Results shown are representative of at least three determinations; variation was 5 to $10 \%$.

Fig. 4. Effect of glyoxylate $(\bullet)$ and pyruvate $(O)$ on $\mathrm{NADP}^{+}$-dependent GDH. Activity was assayed in vitro. The activity corresponding to $100 \%$ was $5.9 \mathrm{U}$ (mg protein $)^{-1}$. Results shown are representative of at least three determinations; variation was 5 to $10 \%$.

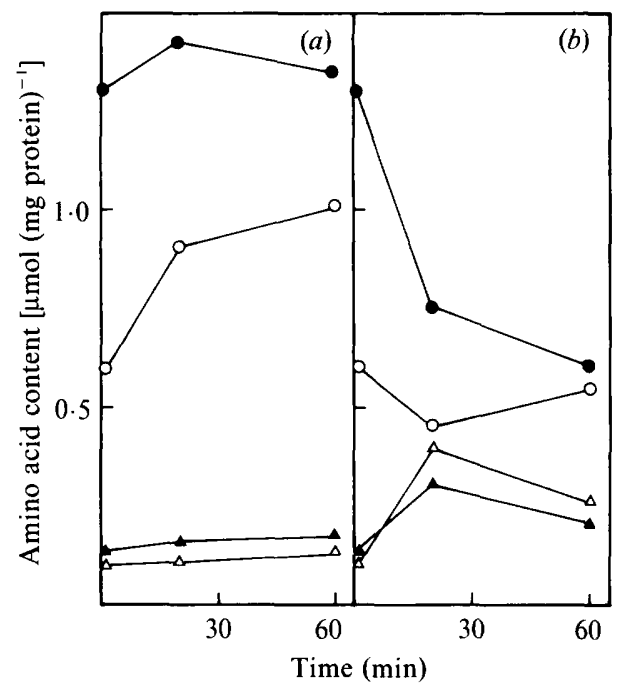

Fig. 5. Effect of glyoxylate on amino acid pools. Cells were grown in MM for $2 \mathrm{~h}$; the culture was then divided and $20 \mathrm{mM}$-glyoxylate was added to one half $(a)$; the rest of the culture was left untreated $(b)$. Amino acid pools ( $\bullet$. glutamate; $O$, glutamine; $\boldsymbol{\Delta}$, alanine; $\triangle$, glycine) were then determined. Results shown are representative of at least three determinations; variation was 5 to $10 \%$.

grown cells (Gosling \& Duggan, 1971). It has also been suggested (Bogonez et al., 1985) that under certain physiological conditions, changes in the NADP+-dependent GDH activity provide control over the rate of 2-oxoglutarate utilization, resulting in the maintenance of a minimum pool of this 2-oxoacid. It is worth noting that strain S288C grew equally well with or without glyoxylate (data not shown), indicating that carbon and nitrogen flows were being coordinately regulated, thus allowing the maintenance of the same growth rate. 


\section{Effect of glyoxylate on nitrogen distribution}

Amino acid analyses were done on glyoxylate-treated and untreated cells. The glutamate pool decreased very rapidly after glyoxylate addition, whereas in MM it remained almost constant (Fig. 5). At the same time, the alanine and glycine pools rose in the presence of glyoxylate. These results suggest that the glutamate amino nitrogen is being distributed through transamination. Furthermore, in glyoxylate-treated cells, the intracellular concentration of glutamine decreased and then increased slightly (Fig. $5 b$ ), suggesting that the increases in alanine and glycine pools could also be due to the functioning of glutamine transaminase, since this enzyme uses pyruvate and glyoxylate as substrates (Soberón \& González, 1987). These results indicate that high intracellular concentrations of 2-oxoacids cause channelling of glutamate and glutamine towards transamination. This results in a decreased glutamine pool which allows glutamine synthetase activity to increase (Fig. 3) and increases ammonium assimilation through the glutamine synthetase-glutamate synthase pathway (Tempest et al., 1970). As judged by amino acid accumulation and growth, total ammonium assimilation was not diminished in glyoxylatetreated cultures (Fig. 5). Our results indicate that the decrease in NADP+-dependent GDH activity is due to the inhibitory effect of glyoxylate and that this 2-oxoacid effects coordinate regulation of carbon and nitrogen metabolism.

The authors are grateful to Gloria Soberón, Irene Castaño and David Romero for their critical review of the manuscript, to Jaime Mora, Guillermo Dávila and Edgardo Escamilla for their enthusiasm and criticism throughout this work, and to Lucila Lulo for secretarial assistance. This work was supported in part by a grant from Fondo de Estudios e Investigación Ricardo J. Zevada.

\section{REFERENCES}

Bergmeyer, M. J. (1963). Methods of Enzymatic Analysis, pp. 253-259. New York \& London: Academic Press.

Bogonez, E., Satrustegi, J. \& Machado, A. (1985). Regulation by ammonium of glutamate dehydrogenase $\left(\mathrm{NADP}^{+}\right)$from Saccharomyces cerevisiae. Journal of General Microbiology 131, 1425-1432.

Dubois, E., Grenson, M. \& Wiame, J. M. (1974). The participation of the anabolic glutamate dehydrogenase in the nitrogen catabolite repression of arginase in Saccharomyces cerevisiae. European Journal of Biochemistry 48, 603-616.

DOHERTY, D. (1970). L-Glutamate dehydrogenases (yeast). Methods in Enzymology 17, 850-856.

FERGUSON, A. R. \& SimS, A. P. (1974). The regulation of glutamine metabolism in Candida utilis : the role of glutamine in the control of glutamine synthetase. Journal of General Microbiology 80, 159-171.

González, A., Tenorio, M., Vaca, G. \& Mora, J. (1983). Neurospora crassa mutant impaired in glutamine regulation. Journal of Bacteriology 155, 1-7.

González, A., Rodríguez, L., Olivera, H. \& SOBERón, M. (1985). NADP+-dependent glutamate dehydrogenase activity is impaired in mutants of Saccharomyces cerevisiae that lack aconitase. Journal of General Microbiology 131, 2565-2571.

Gosling, J. P. \& DUgGan, P. F. (1971). Activities of tricarboxylic acid cycle enzymes, glyoxylate cycle enzymes, and fructose diphosphatase in bakers' yeast during adaptation to acetate oxidation. Journal of Bacteriology 106, 908-914.
Holzer, H. \& SCHNEIDER, S. (1957). Anreicherung und Trennung einer DPN-spezifischen und einer TPN-spezifischen Glutaminosaure Dehydrogenase aus Hefe. Biochemische Zeitschrift 329, 361-367.

Kang, L., Keeler, M., Dunlop, P. C. \& Roon, R. J. (1982). Nitrogen catabolite repression in a glutamate auxothoph of Saccharomyces cerevisiae. Journal of Bacteriology 151, 29-35.

KoRnBERG, H. L. (1966). Anaplerotic sequences and their role in metabolism. Essays in Biochemistry 2,131 .

Parvin, R. (1969). Citrate synthase from yeast. Methods in Enzymology 13, 16-19.

Soberón, M. \& González, A. (1987). Glutamine degradation through the $\omega$-amidase pathway in Saccharomyces cerevisiae. Journal of General Microbiology 133, 9-14.

T ACHIKI, T., Toboro, K., Horino, I. \& Tochiburo, T. (1981). Assimilation of ammonia by Gluconobacter suboxydans through glutamine synthetase/glutamate synthase pathway. Agricultural and Biological Chemistry 45, 1715-1717.

Tempest, D. W., Meers, J. L. \& Brown, C. M. (1970). Synthesis of glutamate in Aerobacter aerogenes by a hitherto unknown route. Biochemical Journal 117, 405-407.

Tribels, F. \& Vogel, G. A. (1966). Degradation of allantoin by Pseudomonas acidovarans. Biochimica et biophysica acta 113, 292-320. 